\title{
Comparison of the AIMS65 Score with Other Risk Stratification Scores in Upper Variceal and Nonvariceal Gastrointestinal Bleeding
}

\author{
Gonçalo Alexandrino, Rita Carvalho, and Jorge Reis \\ Gastroenterology Department, Hospital Prof. Doutor Fernando Fonseca, Amadora, Portugal
}

\section{To the Editor:}

The authors read with interest the article about the performance of three risk stratifying scores in patients with upper gastrointestinal bleeding (UGIB) applied in a Korean population, by Choe et al., ${ }^{1}$ recently published in Gut and Liver.

UGIB is one of the most common emergencies for gastroenterologists, and, as previously discussed in the mentioned article, early risk stratification is important to determine priorities and define which patients benefit from early endoscopy and more aggressive clinical intervention. Although there are risk scores to predict clinical outcomes in UGIB, the most used being the Glasgow-Blatchford score (GBS), Rockall score (RS), and AIMS65 score (AIMS65), uncertainty still exists about the optimal score that could be uniformly applied in clinical practice. In addition, because most studies about risk scoring excluded patients with variceal bleeding, few data are available on the use of this scores in patients with variceal bleeding.

We recently conducted a retrospective single-center study which compared the performance of three scoring systems (GBS, RS, and AIMS65) in Portuguese patients. The consecutive patients admitted to our institution's emergency department with UGIB over a period of 12 months (between January 2016 and December 2016) were included. Both patients with variceal and nonvariceal bleeding were included. Two major endpoints were considered: (1) composite endpoint-need for any clinical intervention (transfusion, endoscopic therapy, surgery or radiological intervention) and (2) UGIB related mortality at 30 days after admission. The accuracy of each scoring system for the considered endpoints was calculated using the area under the receiver operating characteristic curve (AUROC), with 95\% confidence interval. Statistical analysis was performed on MedCalc ${ }^{\circledR}$ statistical software (Medcalc Software, Mariakerke, Belgium).

A total of 202 patients were included, the majority (75\%)
Table 1. Characteristics and Clinical Outcomes of the Study Population $(\mathrm{n}=202)$

\begin{tabular}{|c|c|}
\hline Variable & Value \\
\hline Age, yr & $67(31-94)$ \\
\hline Male sex & $151(75)$ \\
\hline \multicolumn{2}{|l|}{ Comorbidities } \\
\hline Heart failure & $38(19)$ \\
\hline Chronic liver disease & $55(27)$ \\
\hline Chronic kidney disease & $20(10)$ \\
\hline Metastatic malignancy & $24(12)$ \\
\hline \multicolumn{2}{|l|}{ Endoscopic findings } \\
\hline None & $12(6)$ \\
\hline Erosive disease $\mathrm{e}^{*}$ & $32(16)$ \\
\hline Gastric or duodenal ulcer & $75(37)$ \\
\hline Variceal bleeding & $49(24)$ \\
\hline Malignancy of the upper GI tract & $14(7)$ \\
\hline Dieulafoy & $6(3)$ \\
\hline Mallory-Weiss tear & $6(3)$ \\
\hline Gastric/duodenal angiodysplasia & $8(4)$ \\
\hline Mean time of hospital stay, day & $8.9(0-32)$ \\
\hline \multicolumn{2}{|l|}{ Treatments } \\
\hline Blood transfusion & $93(46)$ \\
\hline Endoscopic treatment & $101(50)$ \\
\hline Surgery & $12(6)$ \\
\hline \multicolumn{2}{|l|}{ Outcome } \\
\hline 30-Day mortality & $14(7)$ \\
\hline \multicolumn{2}{|l|}{ Mean score } \\
\hline Glasgow-Blatchford & $10.1(0-20)$ \\
\hline AIMS65 & $1.5(0-4)$ \\
\hline Rockall & $5.8(0-11)$ \\
\hline
\end{tabular}

Data are presented as mean (range) or number (\%).

GI, gastrointestinal.

*Esophagitis, gastritis, and duodenitis.

Correspondence to: Gonçalo Alexandrino

Gastroenterology Department, Hospital Prof. Doutor Fernando Fonseca, IC 19, Amadora 2720-276, Portugal

Tel: +351-966199616, Fax: +351-214345566, E-mail: goncaloalexandrino@gmail.com

Received on August 18, 2017. Accepted on September 19, 2017.

pISSN 1976-2283 eISSN 2005-1212 https://doi.org/10.5009/gnl17380

@) This is an Open Access article distributed under the terms of the Creative Commons Attribution Non-Commercial License (http://creativecommons.org/licenses/by-nc/4.0) which permits unrestricted non-commercial use, distribution, and reproduction in any medium, provided the original work is properly cited. 
being male, with a mean age of 67 years. The mean time from admission to endoscopy was 15.8 hours. The main endoscopic findings were peptic ulcer disease (37\%), variceal bleeding (24\%) and erosive disease (16\%). Six percent of patients had normal endoscopy. The overall rate of 30-day mortality directly related to the UGIB was 7\%. The characteristics and the outcomes of the series of patients are described in Table 1. For the composite endpoint (need for clinical intervention), the AUROCs of GBS, RS, and AIMS65 were, respectively, 0.833, 0.781 and 0.636 in nonvariceal bleeding versus $0.630,0.543$ and 0.620 in variceal bleeding (Figs 1 and 2). For 30-day mortality, the AUROCs of RS, AIMS65 and GBS were, respectively, 0.864, 0.822 and 0.807 in nonvariceal bleeding and 0.783, 0.826 and 0.793 in variceal bleeding (Figs 3 and 4).

GBS was the only effective score to predict the need for clinical intervention, but in our population that was only true for

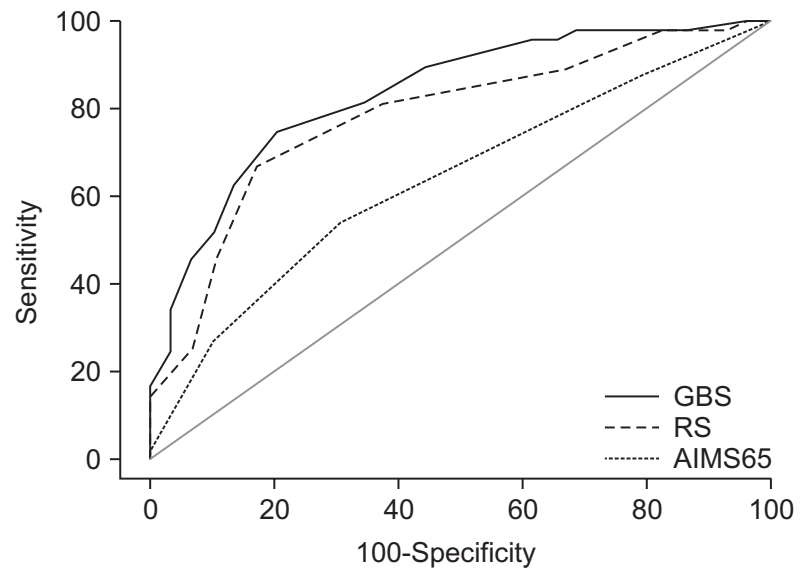

Fig. 1. Area under the receiver operating characteristic curve (AUROC) of Glasgow-Blatchford score (GBS), Rockall score (RS), and AIMS65 score (AIMS65) in predicting need for clinical intervention in nonvariceal upper gastrointestinal bleeding.

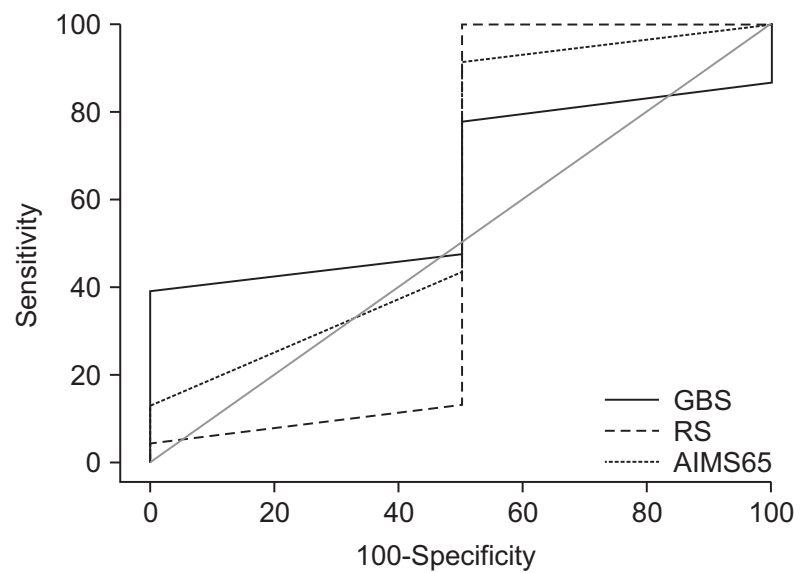

Fig. 2. Area under the receiver operating characteristic curve (AUROC) of Glasgow-Blatchford score (GBS), Rockall score (RS), and AIMS65 score (AIMS65) in predicting need for clinical intervention in variceal upper gastrointestinal bleeding. patients with nonvariceal bleeding. Regarding mortality, the three scores showed a reasonable performance for both variceal and nonvariceal bleeding, but AIMS65 outperformed GBS.

As in the Korean study, GBS seems superior in its ability to predict need for clinical intervention in the nonvariceal bleeding group. Although we had a similar percentage of patients with variceal bleeding as the Korean study, we could not conclude the same in that specific group of patients. GBS seems the best score to predict need for clinical intervention in patients with nonvariceal bleeding, but we would not recommend its use in the variceal group in the Portuguese population.

As in the Korean population, AIMS65 does not seem good to predict clinical intervention in patients admitted with UGIB in the Portuguese population. However, it outperformed GBS and seems a good score to predict mortality both in the nonvariceal and variceal bleeding patients. In fact, AIMS65 was designed to

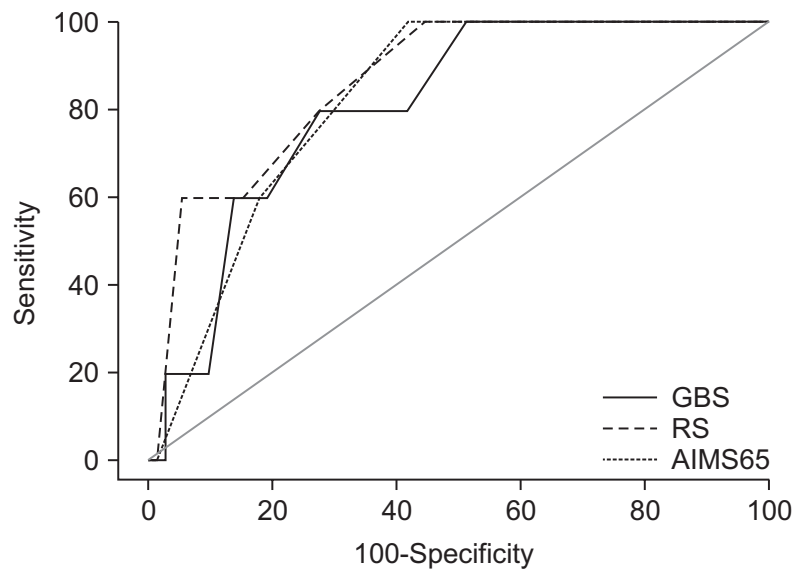

Fig. 3. Area under the receiver operating characteristic curve (AUROC) of Glasgow-Blatchford score (GBS), Rockall score (RS), and AIMS65 score (AIMS65) in predicting 30-day mortality in nonvariceal upper gastrointestinal bleeding.

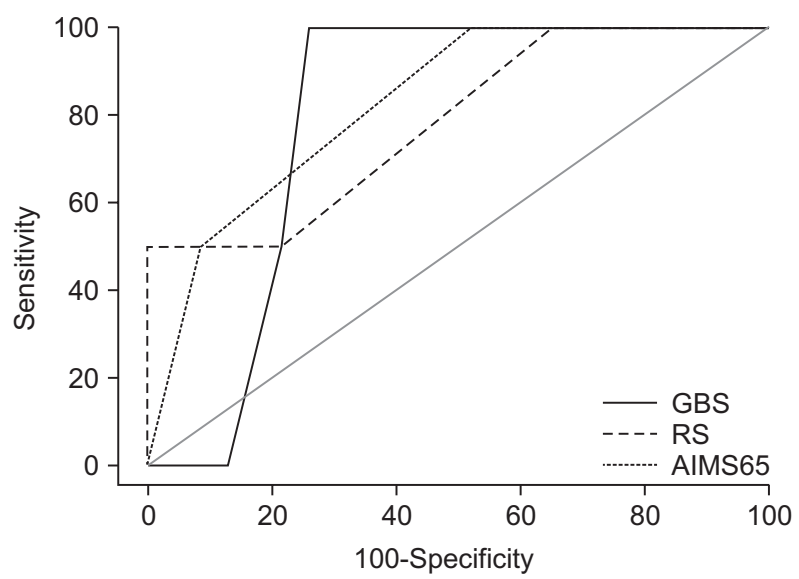

Fig. 4. Area under the receiver operating characteristic curve (AUROC) of Glasgow-Blatchford score (GBS), Rockall score (RS), and AIMS65 score (AIMS65) in predicting 30-day mortality in variceal upper gastrointestinal bleeding. 
determine predictors of mortality. ${ }^{2}$ We should keep in mind that each score was developed to determine a specific, and sometimes different, outcome. Regarding RS, contrary to GBS and AIMS65, it requires endoscopic features, so it is not useful to stratify patients at admission. ${ }^{3}$

In conclusion, in the Portuguese population, GBS seems the best score that predicts need for clinical intervention, but only in patients with nonvariceal UGIB. Although the AIMS65 score is not optimal at predicting clinical intervention, it seems a good at predicting mortality, both in the nonvariceal and variceal UGIB Portuguese population. Although these scores systems are helpful to stratify UGIB patients, its application to different populations should be used with caution, since the causes of UGIB differ considerably among countries. ${ }^{1}$ An ideal scoring system that is uniformly and universally appropriate in clinical practice is yet to be defined.

\section{CONFLICTS OF INTEREST}

No potential conflict of interest relevant to this article was reported.

\section{REFERENCES}

1. Choe JW, Kim SY, Hyun JJ, et al. Is the AIMS 65 score useful in prepdicting clinical outcomes in Korean patients with variceal and nonvariceal upper gastrointestinal bleeding? Gut Liver 2017;11:813-820.

2. Saltzman JR, Tabak YP, Hyett BH, Sun X, Travis AC, Johannes RS. A simple risk score accurately predicts in-hospital mortality, length of stay, and cost in acute upper GI bleeding. Gastrointest Endosc 2011;74:1215-1224.

3. Rockall TA, Logan RF, Devlin HB, Northfield TC. Risk assessment after acute upper gastrointestinal haemorrhage. Gut 1996;38:316321. 